\title{
Droplet Formation at Microfluidic T-junctions
}

Yu Xiang ${ }^{1}$ and David A. LaVan²*

${ }^{1}$ McKinsey \& Company, New York, NY USA

${ }^{2}$ Ceramics Division, Materials Science and Engineering Laboratory, National Institute of Standards and Technology, Gaithersburg, MD 20899, USA

* david.lavan@nist.gov

\section{ABSTRACT}

Analysis of droplet formation in microfluidic systems is important to understand the operation of these devices, and to permit optimal design and process control. Droplet formation in microfluidic Tjunction devices was studied using experimental and numerical methods. The simulations agree well with experimental data from PDMS devices; they show that droplet pinch-off is controlled not by viscous stress, but rather caused by pressure buildup after channel blocking due to the second phase. The period of droplet formation is dependent on velocity of the flow, but not viscosity or interface tension of the fluids. Analysis using dimensionless period, which is equivalent to dimensionless droplet length, shows that dimensionless period is controlled primarily by water fraction but is also dependent on velocity following a power-law relationship. Higher values of capillary number tend to extend the distance for droplet pinch-off. Droplet length does depend on flow velocity at low velocities, but reaches a relatively constant length at higher flow velocities. The coefficient of variation of droplet volume/length increases with increasing capillary number.

\section{INTRODUCTION}

Microfluidic devices have been recognized for a tantalizing potential to provide novel synthesis and research tools[1,2]. Multiphase flows at the micro scale, particularly droplet-based microfluidics, have been of particular interest[3-6]. There are two major techniques reported to generate micro droplets in microfluidic channels: flow-focusing[7, 8] and T-junctions[9-11].

Droplet generation at $\mathrm{T}$-junctions and the underlying mechanism have been carefully investigated. Many believe that the balance between capillary force and viscous force controls the formation of droplets[9-13]. It has been argued that viscous force in such microfluidic devices is too weak to break up droplets[14]. Instead, analytical models of the droplet formation based on simplified geometric assumptions were proposed[14, 15]. Recently, Stone and coworkers[16] showed experimentally that the buildup of pressure in the oil phase due to blocking of the channel by the intruding phase is indeed the major force to compete with interfacial tension. They predicted that droplet size is a linear function of the flow rate ratio.

It is difficult to simulate multi-phase flows, especially to track moving interfaces and deforming topology[17]. Recently, Osher and coworkers[18, 19] have reported a novel method employing a level set function to track the motion of moving boundaries or interfaces. Zhou et al.[20] reported simulations of the generation of droplets in axisymmetric microfluidic flow-focusing devices using this method with adaptive meshing. Microfluidic devices were made from PDMS for testing. A numerical model of the device was created using a conservative form[21] of the level set method using Comsol Multiphysics software. Further numerical details are in the supplemental materials, along with details of the experimental methods used.

Channel geometry can not only affect the production of droplets, but even change the underlying mechanism. For example, the width ratio of the branch and main channel $D_{\text {branch }} / D_{0}$ in a $\mathrm{T}$ junction device[16], the aspect ratio of channel cross-section[16] and the angle of the T junction[23], have been reported to alter the properties of multi-phase flows. In the $2 \mathrm{D}$ numerical model (corresponding to the top-view of the fabricated device), we chose to work with geometry matching the fabricated devices: width, $D_{\text {branch }}=D_{0}=100 \mu \mathrm{m}$ and height, $h=100 \mu \mathrm{m}$. Unless otherwise mentioned, the density of the oil 
phase was $1.7 \times 10^{3} \mathrm{~kg} \mathrm{~m}^{-3}$, density of the water phase, $1.0 \times 10^{3} \mathrm{~kg} \mathrm{~m}^{-3}$; viscosity of the oil phase $21.1 \times 10^{-3}$ $\mathrm{Pa} \cdot \mathrm{s}$, viscosity of the water phase $1.0 \times 10^{-3} \mathrm{~Pa} \cdot \mathrm{s}$; interfacial tension of oil $/$ water $1.2 \times 10^{-3} \mathrm{~N} / \mathrm{m}^{1}$. The contact angle of water on the oil-wetted wall has been shown to have very weak influence on the size of droplet[24]; the contact angle was measured as 144 degrees.

\section{RESULTS and DISCUSSION}

\section{Mechanism of droplet formation}

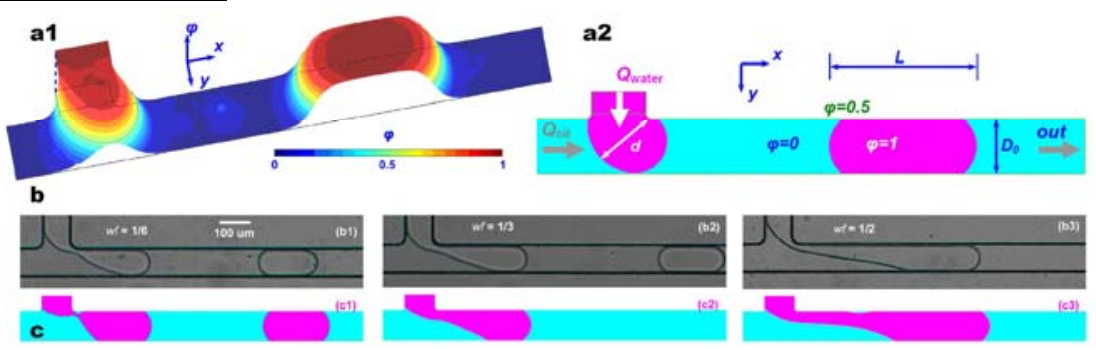

Figure 1. Model and experimental results for droplet formation

The underlying mechanism of droplet formation is controlled by the balance of the hydraulic pressure drop across the interface, $\Delta p$, the viscous stress, $\tau$, and the interfacial tension, $\Omega$ [25].

$$
\Delta p+\tau \equiv \Omega,
$$

Interfacial tension stabilizes a developing droplet and prevents detachment of the droplet from the water stream. Both viscous stress and pressure drop try to snap droplets off from the water stream. Figure 1(b) and (c) present photographs taken during experiments and corresponding simulation results.

Several dimensionless numbers can be employed to describe the formation of droplets. Capillary number, $C a \equiv U \mu / \sigma$, the relative value of viscous stress compared to interfacial tension, depends on the velocity of the carrier fluid flow, $U$, the interfacial tension coefficient at the water/oil interface, $\sigma$, and the dynamic viscosity of the carrier fluid, $\mu$. In this study, experiments were conducted at very low $C a$ values $\left(\mathrm{Ca} \sim 10^{-3}\right)$. $\left(U \sim 10^{-3} \mathrm{~m} / \mathrm{s}, \mu \sim 10^{-3} \mathrm{~Pa} \cdot \mathrm{s}\right.$, and $\left.\sigma \sim 10^{-3} \mathrm{~N} / \mathrm{m}\right)$. At such low $\mathrm{Ca}$ values, the viscous stress is extremely low; it is the hydraulic pressure difference that dominates the left side of the balance equation (1), and competes with the interfacial tension to generate discrete droplets in channels.

Water fraction, $w f$, is defined as the ratio of flow rate of the aqueous phase to the total flow rate of the carrier fluid and the aqueous phase[10], or, $w f \equiv Q_{\text {water }} /\left(Q_{\text {water }}+Q_{\text {oil }}\right)$, where $Q_{\text {water }}$ is the volumetric flow rate of aqueous phase and $Q_{\text {oil }}$ the volumetric flow rate of the carrier fluid (oil) stream. The ratio of flow rates is defined by $\Gamma=Q_{\text {water }} / Q_{\text {oil }}$, or, $\Gamma=w f /(1-w f)$.

Droplet volume, $V$, is proportional to its end-to-end linear length $L$, neglecting a small volume associated with the curvature on the ends of the droplets. To evaluate the variation in size of droplets, simulations of the formation of as many as nine droplets in series were performed at different flow rates, water fractions, interfacial tensions and viscosities (data shown in Table I)

Table I. Simulation results for the formation of a series of droplets

\begin{tabular}{|c|c|c|c|c|c|c|c|c|}
\hline $\begin{array}{l}\text { Carrier } \\
\text { Fluid (oil) }\end{array}$ & $\mathrm{Ca}$ & $\begin{array}{c}U_{\text {total }} \\
(\mathrm{mm} / \mathrm{s})\end{array}$ & $w f$ & $\begin{array}{c}\mathrm{N}: \text { \# of } \\
\text { droplets } \\
\text { simulated }\end{array}$ & $\begin{array}{l}\text { Pinch-off } \\
\text { length } \\
\left(10^{-4} \mathrm{~m}\right)\end{array}$ & $\begin{array}{l}\text { Period of } \\
\text { droplet } \\
\text { formation } \\
\text { (s) }\end{array}$ & $\begin{array}{c}\text { Length of } \\
\text { droplets } \\
\left(10^{-4} \mathrm{~m}\right)\end{array}$ & $\begin{array}{l}\text { Volume of } \\
\text { droplets } \\
(n L)\end{array}$ \\
\hline $\begin{array}{l}\text { Perfluorohe- } \\
\text { xylethanol }\end{array}$ & $\begin{array}{l}8.75 \\
\times 10^{-3}\end{array}$ & 0.75 & 0.333 & 9 & $\begin{array}{c}0.383 \pm \\
0.050\end{array}$ & $\begin{array}{c}0.823 \pm \\
0.096\end{array}$ & $\begin{array}{c}2.924 \pm \\
0.293\end{array}$ & $\begin{array}{c}2.770 \pm \\
0.293\end{array}$ \\
\hline Perfluoro- & 3.64 & 50 & 0.200 & 6 & $0.622 \pm$ & 0.00518 & $1.126 \pm$ & $0.262 \pm$ \\
\hline
\end{tabular}




$\begin{array}{ccccccccc}\text { decaline }^{*} & \mathrm{x} 10^{-3} & & & & 0.064 & \pm 0.000756 & 0.072 & 0.018 \\ & & & & & & & & \\ \text { Perfluoro- } & 1.46 & 20 & 0.200 & 6 & 0.542 \pm & 0.0171 & 1.268 \pm & 0.294 \pm \\ \text { decaline }^{*} & \mathrm{x} 10^{-3} & & & & 0.086 & \pm 0.00197 & 0.064 & 0.0083 \\ \text { Hexadecane } & 6.00 & & & & & & & \\ & \mathrm{x} 10^{-5} & 1.5 & 0.667 & 6 & 0.145 \pm & 0.486 \pm & 5.525 \pm & 5.371 \pm \\ & & & & & 0.012 & 0.021 & 0.261 & 0.261\end{array}$

*Devices have a channel width of $D_{0}=50 \mu \mathrm{m}$ instead of $100 \mu m$.

Figure 2 shows the coefficient of variation, $C V=s t$. dev. / mean, of droplet volume as a function of $\mathrm{Ca}$. At larger $\mathrm{Ca}$, viscous stress becomes more significant compared to interfacial tension, although the growth is still governed by the pressure difference. The increase in the viscous effect alters the uniformity of droplet size. This trend had been reported by Tice et al, whose experiments showed a higher variation of droplet length at higher total flow rate[10].

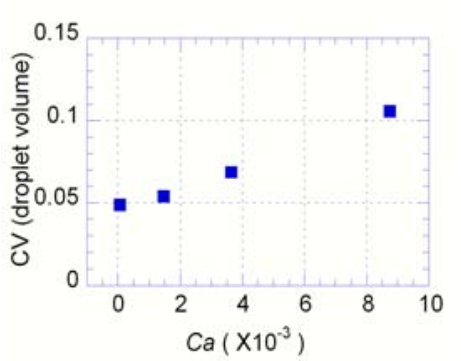

Figure 2. Coefficient of variation of droplet volume as a function of Ca.

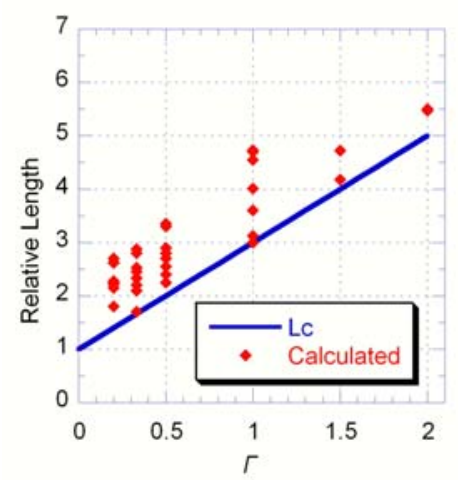

Figure 3. Relative length as a function of flow rate ratio.

The growth of a droplet starts when the water stream enters the main channel, and ends at the pinch-off of a droplet from the stream. This process is composed of two steps: elongation and detachment, $L=$ $L_{\text {elongation }}+L_{\text {detach }}$. The growth rate during elongation is determined mainly by $U_{\text {water }}$. $L_{\text {elongation, }}$ or the intrusion length of water into oil, is often of the same order of magnitude of the width of the main channel, $L_{\text {elongation }} \sim O\left(D_{0}\right)$.

A stable aqueous droplet in oil phase at equilibrium sees a higher hydraulic pressure inside of the droplet than outside. The pressure difference across the interface is the difference in pressure between the oil and water phase, $\Delta p=p_{\text {water }}-p_{\text {oil }}$, and must be balanced by the interfacial tension:

$$
\Delta p=p_{\text {water }}-p_{\text {oil }}=\Omega
$$

At a T-junction, once the water phase blocks the channel, oil can only flow through the thin wetting layer between water and the channel wall; pressure in the oil phase will quickly build up and reduce $\Delta p$; which leads to an imbalance with interfacial tension, resulting in the squeezing of the neck of the water thread \{with a neck width of $d$, as denoted in Figure 1(a2)\}, until the neck breaks off and a droplet detaches.

Negative hydraulic pressure is present near the pinch-off point at the moment of break-off, as shown in Fig. 3a, causing oil from the surrounding area to quickly fill the void. The rate of squeezing is estimated by $U_{\text {oil }}$; the time is $\Delta t_{\text {detach }}=d / U_{\text {oil }}$; the droplet length grows at a rate determined by flow velocity of water, or $L_{\text {detach }}=$ $U_{\text {water }} \cdot \Delta t_{\text {detach }}=d \cdot \Gamma$. Combined with an estimation of $L_{\text {elongation }}$ being $D_{0}$, it leads to an estimate of the total length of a droplet [16], of $L=D_{0}+d \cdot \Gamma$ which can be rewritten as $\widetilde{L}=1+\alpha \Gamma$, where $\widetilde{L}$ is droplet length relative to the channel width $D_{0}$, and $\alpha$ is the ratio of $d / D_{0}$. The droplet size is a linear function of the flow rate ratio, with a constant $\alpha$ determined by device geometry. Figure 3 shows the calculated relative droplet length at different flow rate ratios $\Gamma$, when $C a$ values are kept low (smaller than $10^{-2}$ ). The general linear trend is clearly seen. The value of $\alpha=2$, is in agreement with Garstecki et al.[16] Data at the same $\Gamma$ value were calculated at different $U_{\text {water }}$ levels. As shown in Figure 4, as $U_{\text {water }}$ increases, the droplet length quickly decays and approaches a characteristic droplet length, $L_{c}$, which is only a function of $\Gamma$. The linear fit in Fig.4 is, in fact, the $L_{c}(I)$ function. 


\section{Period of droplet formation}

Droplet formation period increases with water fraction, at a given capillary number, due to the conservation of mass. Increasing $\mathrm{Ca}$ (by increasing flow velocity) will shorten the period; we have examined the parameters of $\mathrm{Ca}$ individually: Fig 5(a) shows that changes in the viscosity of the oil, the oil/DI interfacial tension or changes in both (data not shown) do not cause significant changes to the period when flow velocity is fixed. The same data is plotted against $\mathrm{Ca}$ in Fig 5(b).

The dependence of period on flow velocity is shown in Figure 6; the period drops quickly as velocity increases (starting below $0.5 \mathrm{~mm} / \mathrm{s}$ ). This relationship was found to follow a power law with an exponent close to $7 / 6$ for the studied geometry. For $w f=1 / 3, T=k_{0} / U_{\text {water }}^{1.169}$ with $R^{2}$ equal to $0.9968(\mathrm{~N}=13)(T$ in sec; $U_{\text {water }}$ in $\left.\mathrm{m} / \mathrm{s}, k_{0}=4.98 \times 10^{-5}\right)$.

Because droplet period is so strongly dependent on flow velocity, changes in velocity will result in

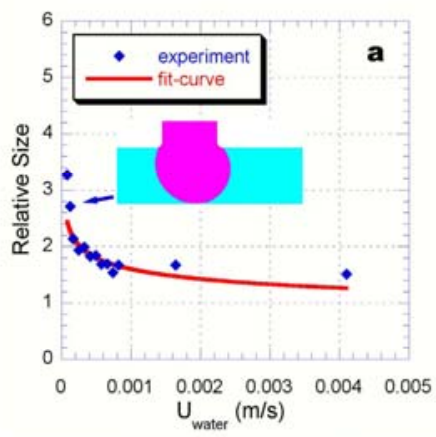
different periods even at the same $C a$. Changes in viscosity and interfacial tension change $C a$ but do not change period. Table II provides results corresponding to flow conditions at the same $w f$ value but at different values of flow velocity, viscosity and interfacial tension.

Period, $T$, is composed of elongation and detachment: $T=\Delta t_{\text {elongation }}$ $+\Delta t_{\text {detach }}$. During elongation, at a low $C a$ and moderate $w f$, the water thread extends almost straight into the oil without significant deformation, until it touches the opposite sidewall. A rough estimate of elongation time can be given by $\Delta t_{\text {elongation }}=D_{0} / U_{\text {water }}$. The detachment

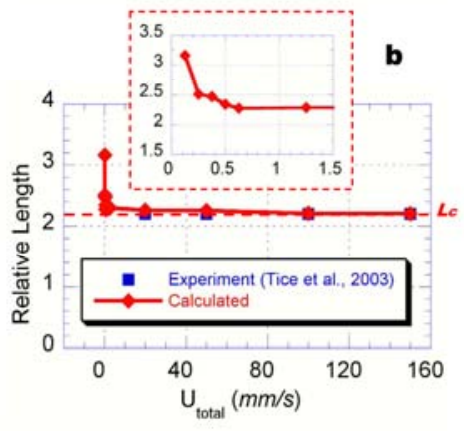

Figure 4. Droplet size and length as a function of velocity time is estimated by $\Delta t_{\text {detach }}=d / U_{\text {oil }}$, relating period to both flow velocity and the ratio of flow rates,

$$
T=\frac{D_{0}}{U_{\text {water }}}\left(1+\frac{d}{D_{0}} \Gamma\right)
$$

If using $D_{0} / U_{\text {water }}$ as the time scale, one can convert $T$ to a dimensionless period, $\widetilde{T}=1+\frac{d}{D_{0}} \Gamma$, which is also the relative length of droplets.

At a given flow rate ratio, $\Gamma$, period, $T$, should be proportional to $1 / U_{\text {water }}$ according to (2); however, at extremely low $U_{\text {water }}$ level when $\Gamma$ is fixed, interfacial tension causes the water thread to expand dramatically during the elongation step, leading $D_{0} / U_{\text {water }}$ to under-estimate $\Delta t_{\text {elongation, }}$, which is why T scales with $U_{\text {water }}^{-\beta}(\beta>1)$ but not with $U_{\text {water }}^{-1}$.

Table II. Calculated periods at different flow conditions

\begin{tabular}{|c|c|c|c|}
\hline Carrier Fluid (Oil) & $\begin{array}{c}(\mathrm{A}) \\
\text { hexadecane }\end{array}$ & $\begin{array}{c}\text { (B) } \\
\text { hexadecane }\end{array}$ & (C) perfluorohexylethanol \\
\hline$\mu$, measured viscosity (oil), (Pa s) & $3 \times 10^{-3}$ & $3 \times 10^{-3}$ & $21.1 \times 10^{-3}$ \\
\hline $\begin{array}{c}\sigma, \text { measured interface tension } \\
(\text { water/oil }),(\mathrm{N} / \mathrm{m})\end{array}$ & $25 \times 10^{-3}$ & $25 \times 10^{-3}$ & $1.2 \times 10^{-3}$ \\
\hline$U_{o i l}$, oil velocity, $(\mathrm{m} / \mathrm{s})$ & $73.2 \times 10^{-3}$ & $0.5 \times 10^{-3}$ & $0.5 \times 10^{-3}$ \\
\hline$U_{\text {water }}$, water velocity, $(\mathrm{m} / \mathrm{s})$ & $36.6 \times 10^{-3}$ & $0.25 \times 10^{-3}$ & $0.25 \times 10^{-3}$ \\
\hline $\mathrm{Ca}$, Capillary Number & $8.79 \times 10^{-3}$ & $6 \times 10^{-5}$ & $8.79 \times 10^{-3}$ \\
\hline$T$, calculated period, (s) & 0.004 & 0.84 & 0.82 \\
\hline
\end{tabular}



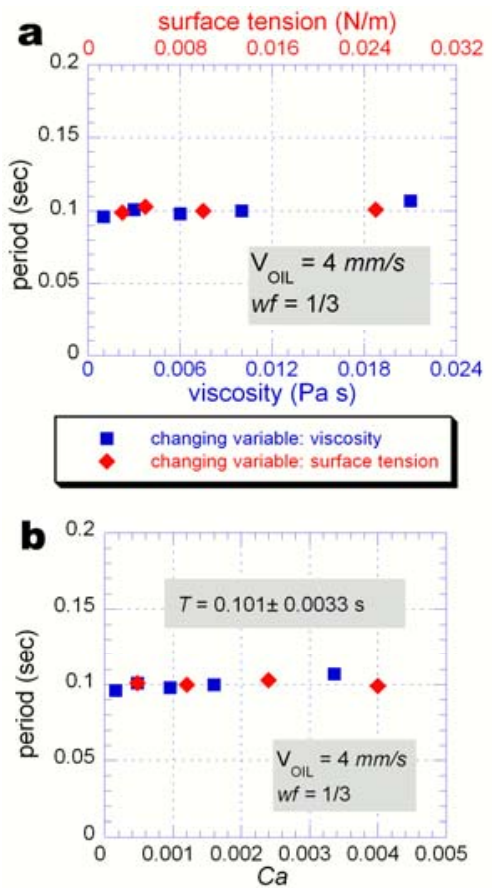

- changing variable: viscosity

- changing variable: surface tension

Figure 5. Period as a function of viscosity and $\mathrm{Ca}$

\section{Length of droplets}

From (2) and (3), it is easy to see $L=U_{\text {water }} \cdot T$. Recall that $T=k_{0} / U_{\text {water }}^{\beta}$ (where $\beta=1.169$ ), so the length of a droplet, $L$, is found to be weakly dependent on water velocity, $L=k_{0} / U_{\text {water }}{ }^{\beta-1}$, in accordance with the experimental droplet length data shown in Fig.4(a). The explanation arises from the invalidity of the assumption that $L_{\text {elongation }}=D_{0}$. At extremely low $U_{\text {water }}$ levels, before the water thread can block the channel, surface tension has already expanded the water thread, approaching a spherical shape, as shown in the inset of Figure 4(a). The equivalent elongation length will be larger than the main channel width, $D_{0}$, which consequently causes $L$ to be larger than the characteristic length $L_{c}=D_{0}+d \cdot \Gamma$. It is still not clear as to why the power law exponent is close to $-0.17\left(-1 / 6^{\text {th }}\right)$. Similar power laws relating $L$ to $U_{\text {water }}$ in similar geometries have been reported, with varying exponents, such as -0.30 by Xu et al.[26] and -0.25 by Van der Graaf et al. [24] The power law relationship also predicts that droplet length is essentially constant once a threshold flow velocity has been reached. Tice et al.[10] reported constant droplet length independent of flow velocity with $U_{\text {water }}$ varying between 4 and $30 \mathrm{~mm} / \mathrm{s}$ at $w f=0.2$; this observation is correct because all these velocities are above the threshold; the difference in droplet length became undetectable. Under similar conditions $\left(D_{0}=50 \mu \mathrm{m}, \sigma=14 \mathrm{mN} / \mathrm{m}, \mu_{O I L}=5.1 \mathrm{mPa} \mathrm{s}\right)$, but at a broader range of velocities, the droplet length varies as shown in Fig. 4(b).

\section{CONCLUSIONS}

The droplet grows at a rate given by $U_{\text {water }}$; in the detachment step, the neck of a developing droplet is squeezed at a rate governed by $U_{\text {oil }}$. The frequency at which the droplets are generated is dependent on water fraction and velocity, but not significantly on viscosity or interfacial tension. The dependence of the period on velocity is described by a power law relationship with exponent of $7 / 6$. The length of droplets varies at low flow rates, and approaches a constant characteristic length associated with a corresponding water fraction. Modeling of droplet fission further proves that pressure buildup due to channel blocking is the major contributing force for droplet break-off.

\section{ACKNOWLEDGMENTS}

The full description of the procedures used in this paper requires the identification of certain software and their suppliers. The inclusion of such information should in no way be construed as indicating that such software is endorsed by NIST or is recommended by NIST or that it is necessarily the best software for the purposes described.

\section{REFERENCES}

[1] G. M. Whitesides, "The origins and the future of microfluidics," Nature (London, United Kingdom), vol. 442, pp. 368-373, 2006.

[2] T. Thorsen, J. Maerkl Sebastian, and R. Quake Stephen, "Microfluidic large-scale integration," Science, vol. 298, pp. 580-4, 2002.

[3] D. R. Link, S. L. Anna, D. A. Weitz, and H. A. Stone, "Geometrically Mediated Breakup of Drops in Microfluidic Devices," Physical Review Letters, vol. 92, pp. 054503/1-054503/4, 2004.

[4] A. Guenther, S. A. Khan, M. Thalmann, F. Trachsel, and K. F. Jensen, "Transport and reaction in microscale segmented gas-liquid flow," Lab on a Chip, vol. 4, pp. 278-286, 2004. 
[5] J. D. Tice, R. F. Ismagilov, and B. Zheng, "Forming droplets in microfluidic channels with alternating composition and application to indexing concentrations in droplet-based assays," Abstracts of Papers, 228th ACS National Meeting, Philadelphia, PA, United States, August 22-26, 2004, pp. CHED-106, 2004.

[6] H. Song, D. L. Chen, and R. F. Ismagilov, "Reactions in droplets in microfluidic channels," Angewandte Chemie, International Edition, vol. 45, pp. 7336-7356, 2006.

[7] P. Garstecki, I. Gitlin, W. DiLuzio, G. M. Whitesides, E. Kumacheva, and H. A. Stone, "Formation of monodisperse bubbles in a microfluidic flow-focusing device," Applied Physics Letters, vol. 85, pp. 26492651, 2004.

[8] S. L. Anna, N. Bontoux, and H. A. Stone, "Formation of dispersions using $\backslash$ "flow focusing $\backslash "$ in microchannels," Applied Physics Letters, vol. 82, pp. 364-366, 2003.

[9] T. Thorsen, R. W. Roberts, F. H. Arnold, and S. R. Quake, "Dynamic Pattern Formation in a VesicleGenerating Microfluidic Device," Physical Review Letters, vol. 86, pp. 4163-4166, 2001.

[10] J. D. Tice, H. Song, A. D. Lyon, and R. F. Ismagilov, "Formation of Droplets and Mixing in Multiphase Microfluidics at Low Values of the Reynolds and the Capillary Numbers," Langmuir, vol. 19, pp. 91279133, 2003.

[11] J. D. Tice, A. D. Lyon, and R. F. Ismagilov, "Effects of viscosity on droplet formation and mixing in microfluidic channels," Analytica Chimica Acta, vol. 507, pp. 73-77, 2004.

[12] V. Cristini and Y.-C. Tan, "Theory and numerical simulation of droplet dynamics in complex flows - a review," Lab on a Chip, vol. 4, pp. 257-264, 2004.

[13] M. D. Menech, "Modeling of droplet breakup in a microfluidic T-shaped junction with a phase-field model," Phys Rev E, vol. 73, pp. 031505, 2006.

[14] P. Guillot and A. Colin, "Stability of parallel flows in a microchannel after a T junction," Physical Review E: Statistical, Nonlinear, and Soft Matter Physics, vol. 72, pp. 066301/1-066301/4, 2005.

[15] J. H. Xu, G. S. Luo, G. G. Chen, and J. D. Wang, "Experimental and theoretical approaches on droplet formation from a micrometer screen hole," Journal of Membrane Science, vol. 266, pp. 121-131, 2005.

[16] P. Garstecki, M. J. Fuerstman, H. A. Stone, and G. M. Whitesides, "Formation of droplets and bubbles in a microfluidic T-junction-scaling and mechanism of break-up," Lab on a Chip, vol. 6, pp. 437-446, 2006.

[17] W. B. J. Zimmerman, Process modelling and simulation with finite element methods. Singapore ; Hackensack, NJ: World Scientific, 2004.

[18] F. Losasso, R. Fedkiw, and S. Osher, "Spatially adaptive techniques for level set methods and incompressible flow," Computers \& Fluids, vol. 35, pp. 995-1010, 2006.

[19] S. Osher and J. Sethian, "Fronts propagating with curvaturedependent speed: algorithms based on Hamilton-Jacobi formulations.," Journal of Computational Physics, vol. 79, pp. 12-49, 1988.

[20] C. Zhou, P. Yue, and J. J. Feng, "Formation of simple and compound drops in microfluidic devices," Physics of Fluids, vol. 18, pp. 092105/1-092105/14, 2006.

[21] E. Olsson and G. Kreiss, "A conservative level set method for two phase flow," Journal of Computational Physics, vol. 210, pp. 225, 2005.

[22] Comsol, Comsol 3.3a Release Notes, 2007.

[23] L. Menetrier-Deremble and P. Tabeling, "Droplet breakup in microfluidic junctions of arbitrary angles," Physical Review E: Statistical, Nonlinear, and Soft Matter Physics, vol. 74, pp. 035303/1-035303/4, 2006.

[24] S. Van der Graaf, T. Nisisako, C. G. P. H. Schroeen, R. G. M. Van der Sman, and R. M. Boom, "Lattice Boltzmann Simulations of Droplet Formation in a T-Shaped Microchannel," Langmuir, vol. 22, pp. 41444152, 2006.

[25] T. Ward, M. Faivre, M. Abkarian, and H. A. Stone, "Microfluidic flow focusing: Drop size and scaling in pressure versus flow-rate-driven pumping," Electrophoresis, vol. 26, pp. 3716-3724, 2005.

[26] J. H. Xu, S. W. Li, J. Tan, Y. J. Wang, and G. S. Luo, "Preparation of highly monodisperse droplet in a Tjunction microfluidic device," AIChE Journal, vol. 52, pp. 3005-3010, 2006. 